Supporting Information for

\title{
Suppression effect of gamma-valerolactone (GVL) on the mild alkaline pretreatment of hybrid pennisetum
}

Yafeng Fan ${ }^{1,2,3,6}$, Lianhua $\mathrm{Li}^{1,2,3 *}$, Gaixiu Yang ${ }^{1,2,3}$, Yongming Sun ${ }^{1,2,3}$, Linsong $\mathrm{He}^{1,2,3}$, Peiwen $\mathrm{Wu}^{1,2,3}$, Chun Ho Lam ${ }^{4}$, Bing Song ${ }^{5 *}$

${ }^{1}$ Guangzhou Institute of Energy Conversion, Chinese Academy of Sciences, Guangzhou 510640, P.R. China

${ }^{2}$ Guangzhou Institute of Energy Conversion, CAS Key Laboratory of Renewable Energy, Chinese Academy of Sciences, Guangzhou 510640, P.R. China.

${ }^{3}$ Guangdong Key Laboratory of New and Renewable Energy Research and Development, Guangzhou 510640, P.R. China.

${ }^{4}$ School of Energy and Environment and State Key Laboratory of Marine Pollution, City

University of Hong Kong, Kowloon Tong, Hong Kong SAR

${ }^{5}$ Scion, Te Papa Tipu Innovation Park, 49 Sala Street, Private Bag 3020, Rotorua 3046, New

Zealand

${ }^{6}$ Nano Science and Technology Institute, University of Science and Technology of China, Suzhou 215123, P.R. China

*Emails of Corresponding Authors:

lilh@ms.giec.ac.cn (Lianhua Li);

bing.song@scionresearch.com (Bing Song).

\section{Contents}

Figure S1 Pictures of liquids obtained after pretreatment and filtration $\ldots \ldots \ldots \ldots \ldots \ldots \ldots . . . . \ldots \ldots$

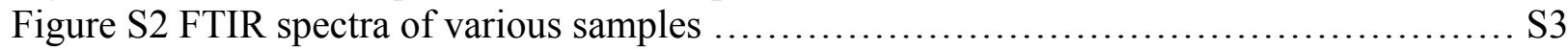

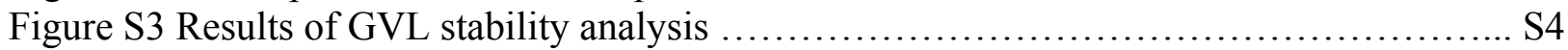

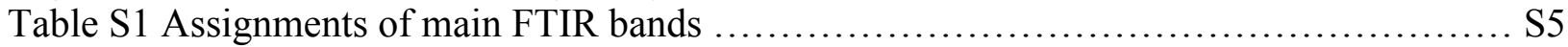

Table S2 Assignments of main NMR signals ............................................ S6

Table S3 Alkalinities of various GVL/NaOH/water mixtures .......................... S7

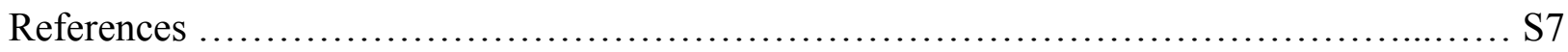

Number of Pages: 7; Number of Figures: 3; Number of Tables: 3 


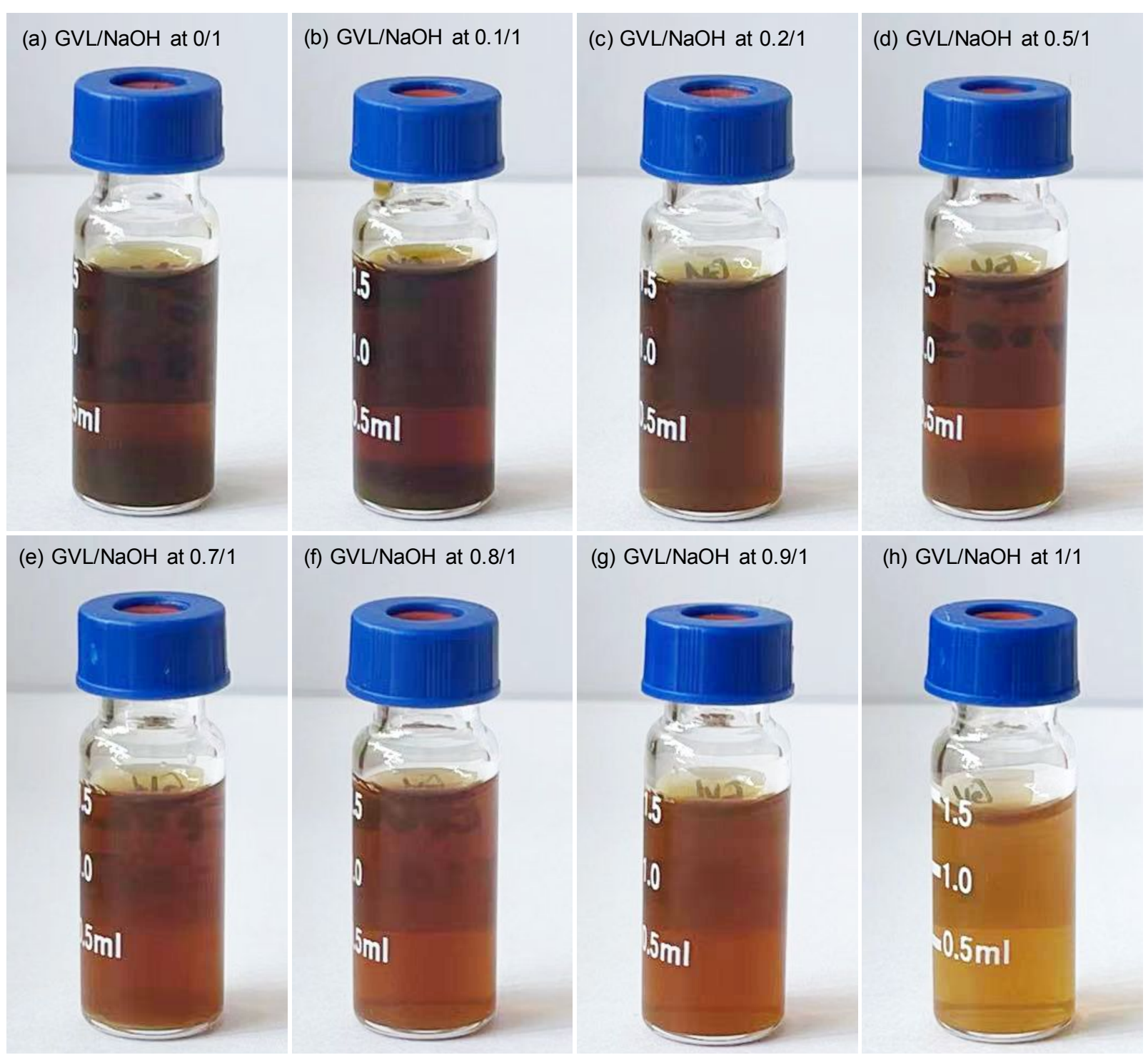

Figure S1. Pictures of liquids obtain from hybrid pennisetum pretreatment by $\mathrm{GVL} / \mathrm{NaOH}$ at different molar ratios (a-h). 


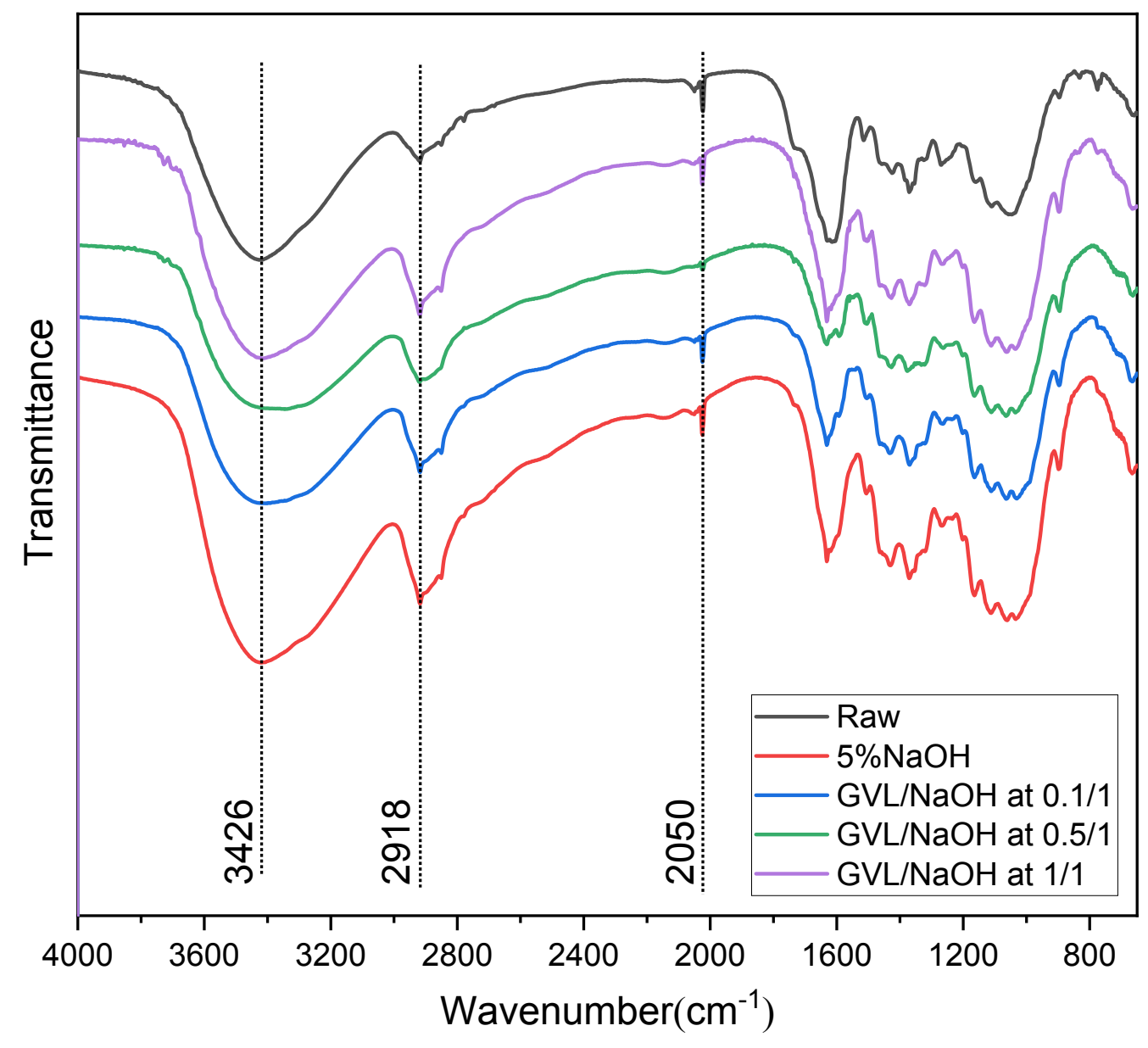

Figure S2. FTIR spectra of raw and pretreated hybrid pennisetum with GVL/NaOH at different molar ratios. 

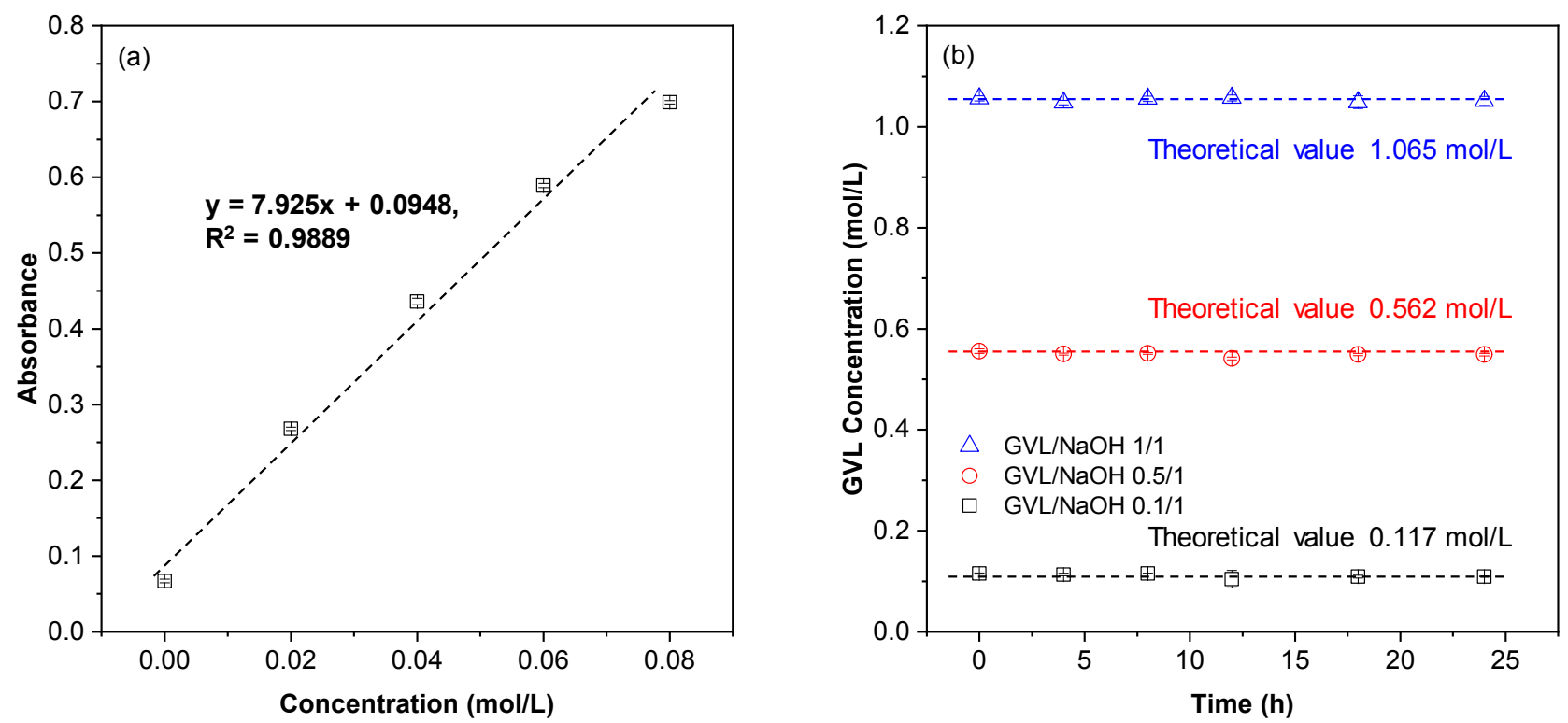

Figure S3. Absorbance of GVL/NaOH/water at the Lambda max $\left(\lambda_{\max }\right)$ wavelength $(224 \mathrm{~nm})$

with GVL from 0 to $0.08 \mathrm{~mol} / \mathrm{L}$ (a) and the time dependent changes of GVL concentrations under $37^{\circ} \mathrm{C}(\mathrm{b})$. 
Table S1. Assignments of main FTIR bands of raw and pretreated hybrid pennisetum samples at the fingerprint region ${ }^{1,2}$

\begin{tabular}{|c|c|}
\hline $\begin{array}{l}\text { absorption } \\
\text { bands }\left(\mathrm{cm}^{-1}\right)\end{array}$ & assignments \\
\hline $\begin{array}{l}1730 \\
1605-1593 \\
1511\end{array}$ & $\begin{array}{l}\mathrm{C}=\mathrm{O} \text { stretch in acetoxy groups }\left(\mathrm{H}_{3} \mathrm{C}-(\mathrm{C}=\mathrm{O})-\mathrm{O}\right) \text { - in hemicellulose } \\
\text { aromatic skeletal vibrations and } \mathrm{C}=\mathrm{O} \text { stretch in lignin } \\
\text { aromatic skeletal vibrations in lignin }\end{array}$ \\
\hline 1464 & $\begin{array}{l}\mathrm{C}-\mathrm{H} \text { deformations asymmetric in } \mathrm{CH}_{3} \text { (in lignin } \mathrm{CH}_{3}-\mathrm{O} \text { and } \\
\left.\left.\text { hemicellulose } \mathrm{CH}_{3}-(\mathrm{C}=\mathrm{O})-\right) \text { and } \mathrm{CH}_{2} \text { (strong in cellulose } \mathrm{I}\right)\end{array}$ \\
\hline 1427 & $\begin{array}{l}\mathrm{CH}_{2} \text { symmetric bending in both crystallized cellulose and } \\
\text { amorphous cellulose }\end{array}$ \\
\hline 1370 & $\begin{array}{l}\mathrm{C}-\mathrm{H} \text { and } \mathrm{CH}_{3} \text { bending vibration in cellulose and hemicelluloses } \\
\text { syringyl ring plus condensed guaiacyl ring; } \mathrm{CH}_{2} \text { in crystallized }\end{array}$ \\
\hline 1319 & $\begin{array}{l}\text { cellulose I (wagging); } \mathrm{C}-\mathrm{H} \text { deformation in cellulose and } \mathrm{C}-\mathrm{O} \\
\text { vibration in syringyl derivatives }\end{array}$ \\
\hline 1264 & $\begin{array}{l}\text { guaiacyl ring plus } \mathrm{C}=\mathrm{O} \text { stretch; } \mathrm{vC}_{\mathrm{ar}}-\mathrm{O} \text { guaiacyl aromatic } \\
\text { methoxyl group in lignin and cellulose }\end{array}$ \\
\hline 1203 & $\mathrm{OH}$ in-plane bending in cellulose I and cellulose II \\
\hline 1157 & $\begin{array}{l}\text { C-O stretch in ester group (conjugated) } \mathrm{C}-\mathrm{O}-\mathrm{C} \text { asymmetric } \\
\text { stretch vibration in cellulose and hemicellulose }\end{array}$ \\
\hline 1114 & $\begin{array}{l}\mathrm{CH} \text { stretching vibrations in different groups of lignin and } \\
\text { cellulose and hemicelluloses }\end{array}$ \\
\hline 1031 & C-O-C skeletal vibration of polysaccharides ring \\
\hline 897 & $\begin{array}{l}\mathrm{C}_{1}-\mathrm{O}-\mathrm{C} \beta-(1-4) \text {-glycosidic linkage (weak and broad in cellulose } \\
\mathrm{I} \text {, strong and sharp in cellulose II) }\end{array}$ \\
\hline 835 & $\begin{array}{l}\text { C-H out of plane bending of 1,2,4-tetrasubstituted aromatic in } \\
\text { positions } 2 \text { and } 6 \text { of } S \text { units, and in all positions of } \mathrm{H} \text { units }\end{array}$ \\
\hline
\end{tabular}


Table S2. Assignments of main ${ }^{13} \mathrm{C}-{ }^{1} \mathrm{H}$ cross-signals in the HSQC spectra of lignin precipitated from the pretreatment solution ${ }^{3-6}$

\begin{tabular}{|c|c|c|}
\hline Lable & $\delta_{\mathrm{C}} / \delta_{\mathrm{H}}(\mathrm{ppm})$ & Assignments \\
\hline$-\mathrm{OCH}_{3}$ & $55.5 / 3.70$ & $\mathrm{C}-\mathrm{H}$ in methoxyls \\
\hline $\mathrm{A}_{\gamma}$ & $59.8-60.8 / 3.37-3.72$ & $\mathrm{C}_{\gamma}-\mathrm{H}_{\gamma}$ in $\beta-O-4^{\prime}$ substructures (A) \\
\hline $\mathrm{X}_{5}$ & $62.6 / 3.40$ and 3.72 & $\mathrm{C}_{5}-\mathrm{H}_{5}$ in $\beta$-D-xylopyranoside (X) \\
\hline $\mathrm{C}_{\gamma}$ & $62.7 / 3.80$ & $\mathrm{C}_{\gamma}-\mathrm{H}_{\gamma}$ in phenylcoumaran substructures $(\mathrm{C})$ \\
\hline $\mathrm{A}_{\alpha}$ & $71.8 / 4.86$ & $\mathrm{C}_{\alpha}-\mathrm{H}_{\alpha}$ in $\beta-O-4^{\prime}$ units (A) \\
\hline $\mathrm{X}_{2}$ & $72.5 / 3.02$ & $\mathrm{C}_{2}-\mathrm{H}_{2}$ in $\beta$-D-xylopyranoside $(\mathrm{X})$ \\
\hline $\mathrm{X}_{3}$ & $73.7 / 3.22$ & $\mathrm{C}_{3}-\mathrm{H}_{3}$ in $\beta$-D-xylopyranoside $(\mathrm{X})$ \\
\hline $\mathrm{X}_{4}$ & $75.4 / 3.60$ & $\mathrm{C}_{4}-\mathrm{H}_{4}$ in $\beta$-D-xylopyranoside $(\mathrm{X})$ \\
\hline $\mathrm{A}_{\beta(\mathrm{G} / \mathrm{H})}$ & $83.9 / 4.29$ & $\mathrm{C}_{\beta}-\mathrm{H}_{\beta}$ in $\beta-O-4^{\prime}$ substructures linked to $\mathrm{G} / \mathrm{H}$ units (A) \\
\hline $\mathrm{B}_{\alpha}$ & $84.7 / 4.64$ & $\mathrm{C}_{\alpha}-\mathrm{H}_{\alpha}$ in $\beta-\beta^{\prime}$ resinol substructures (B) \\
\hline $\mathrm{A}_{\beta(\mathrm{S})}$ & $85.9 / 4.10$ & $\mathrm{C}_{\beta}-\mathrm{H}_{\beta}$ in $\beta-O-4^{\prime}$ substructures linked to $\mathrm{S}$ units (A) \\
\hline $\mathrm{S}_{2,6}$ & $103.8 / 6.71$ & $\mathrm{C}_{2,6}-\mathrm{H}_{2,6}$ in syringyl units $(\mathrm{S})$ \\
\hline $\mathrm{S}_{2,6}^{\prime}$ & $106.2 / 7.23$ & $\mathrm{C}_{2,6}-\mathrm{H}_{2,6}$ in oxidized $\left(\mathrm{C}_{\alpha}=\mathrm{O}\right)$ syringyl units $\left(\mathrm{S}^{\prime}\right)$ \\
\hline $\mathrm{G}_{2}$ & $110.9 / 6.98$ & $\mathrm{C}_{2}-\mathrm{H}_{2}$ in guaiacyl units $(\mathrm{G})$ \\
\hline $\mathrm{G}_{5}$ & $114.9 / 6.77$ & $\mathrm{C}_{5}-\mathrm{H}_{5}$ in guaiacyl units $(\mathrm{G})$ \\
\hline $\mathrm{G}_{6}$ & $119.0 / 6.80$ & $\mathrm{C}_{6}-\mathrm{H}_{6}$ in guaiacyl units $(\mathrm{G})$ \\
\hline $\mathrm{H}_{2,6}$ & 127.9/7.19 & $\mathrm{C}_{2,6}-\mathrm{H}_{2,6}$ in $\mathrm{H}$ units $(\mathrm{H})$ \\
\hline $\mathrm{PCA}_{2,6}$ & $130.1 / 7.48$ & $\mathrm{C}_{2,6}-\mathrm{H}_{2,6}$ in $p$-coumarate substructures (PCA) \\
\hline $\mathrm{FA}_{2}$ & $111.1 / 7.34$ & $\mathrm{C}_{2}-\mathrm{H}_{2}$ in ferulate (FA) \\
\hline $\mathrm{FA}_{6}$ & $122.9 / 7.13$ & $\mathrm{C}_{6}-\mathrm{H}_{6}$ in ferulate $(\mathrm{FA})$ \\
\hline $\mathrm{FA}_{\alpha}$ & $144.7 / 7.46$ & $\mathrm{C}_{\alpha}-\mathrm{H}_{\alpha}$ in ferulate (FA) \\
\hline $\mathrm{PCA}_{\alpha}$ & $144.7 / 7.46$ & $\mathrm{C}_{\alpha}-\mathrm{H}_{\alpha}$ in $p$-coumarate (PCA) \\
\hline
\end{tabular}


Table S3. Alkalinities of various $\mathrm{GVL} / \mathrm{NaOH} /$ water and $\mathrm{NaOH} /$ water mixtures

\begin{tabular}{lccccccc}
\hline \multicolumn{3}{c}{$\mathrm{GVL} / \mathrm{NaOH} /$ water $(5 \% \mathrm{NaOH})$} & \multicolumn{3}{c}{$\mathrm{NaOH} /$ water with different $\mathrm{NaOH}$} \\
\hline $\begin{array}{l}\text { GVL/NaOH } \\
\text { molar ratio }\end{array}$ & $\mathrm{PA}(\mathrm{mg} / \mathrm{L})$ & $\mathrm{IA}(\mathrm{mg} / \mathrm{L})$ & $\mathrm{TA}(\mathrm{mg} / \mathrm{L})$ & $\begin{array}{c}\mathrm{NaOH} \\
\text { content }(\%)\end{array}$ & $\mathrm{PA}(\mathrm{mg} / \mathrm{L})$ & $\mathrm{IA}(\mathrm{mg} / \mathrm{L})$ & $\mathrm{TA}(\mathrm{mg} / \mathrm{L})$ \\
\hline $0 / 1$ & 63793.8 & 893.8 & 64687.5 & $5.00 \%$ & 63793.8 & 893.8 & 64687.5 \\
$0.1 / 1$ & 57531.3 & 3750.0 & 61281.3 & $4.68 \%$ & 63781.3 & 793.8 & 64575.0 \\
$0.2 / 1$ & 51087.5 & 6531.3 & 57618.8 & $4.39 \%$ & 58743.8 & 1712.5 & 60456.3 \\
$0.5 / 1$ & 32937.5 & 14131.3 & 47068.8 & $3.55 \%$ & 47743.8 & 1312.5 & 49056.3 \\
$0.7 / 1$ & 21800.0 & 19131.3 & 40931.3 & $3.06 \%$ & 42831.3 & 506.3 & 43337.5 \\
$0.8 / 1$ & 16750.0 & 19818.8 & 36568.8 & $2.72 \%$ & 36793.8 & 731.3 & 37525.0 \\
$0.9 / 1$ & 10750.0 & 23175.0 & 33925.0 & $2.51 \%$ & 32406.3 & 643.8 & 33050.0 \\
$1 / 1$ & 5662.5 & 25731.3 & 31393.8 & $2.31 \%$ & 32368.8 & 625.0 & 32993.8 \\
\hline
\end{tabular}

\section{Reference}

1. Boukir, A.; Fellak, S.; Doumenq, P., Structural characterization of Argania spinosa Moroccan wooden artifacts during natural degradation progress using infrared spectroscopy (ATR-FTIR) and X-Ray diffraction (XRD). Heliyon 2019, 5 (9), e02477. doi: 10.1016/j.heliyon.2019.e02477

2. Chen, B.; Wang, X.; Leng, W.; Mei, C.; Zhai, S., Spectroscopic/Microscopic elucidation for chemical changes during acid pretreatment on arundo donax. J. Bioresour. Bioprod. 2019, 4 (3), 192-199. doi: 10.12162/jbb.v4i3.008

3. Li, H.-Y.; Wang, B.; Wen, J.-L.; Cao, X.-F.; Sun, S.-N.; Sun, R.-C., Availability of four energy crops assessing by the enzymatic hydrolysis and structural features of lignin before and after hydrothermal treatment. Energy Convers. Manag. 2018, 155, 58-67. doi:

10.1016/j.enconman.2017.10.089

4. Huang, C.; Wang, X.; Liang, C.; Jiang, X.; Yang, G.; Xu, J.; Yong, Q., A sustainable process for procuring biologically active fractions of high-purity xylooligosaccharides and water-soluble lignin from Moso bamboo prehydrolyzate. Biotechnol. biofuels 2019, 12 (1), 1-13. doi: 10.1186/s13068-019-1527-3

5. Wen, J.-L.; Xue, B.-L.; Xu, F.; Sun, R.-C.; Pinkert, A., Unmasking the structural features and property of lignin from bamboo. Ind. Crops Prod. 2013, 42, 332-343. doi:

10.1016/j.indcrop.2012.05.041

6. Jin, C.; Yang, M.; Shuang, E.; Liu, J.; Zhang, S.; Zhang, X.; Sheng, K.; Zhang, X., Corn stover valorization by one-step formic acid fractionation and formylation for 5-

hydroxymethylfurfural and high guaiacyl lignin production. Bioresour. Technol. 2020, 299, 122586. doi: 10.1016/j.biortech.2019.122586 